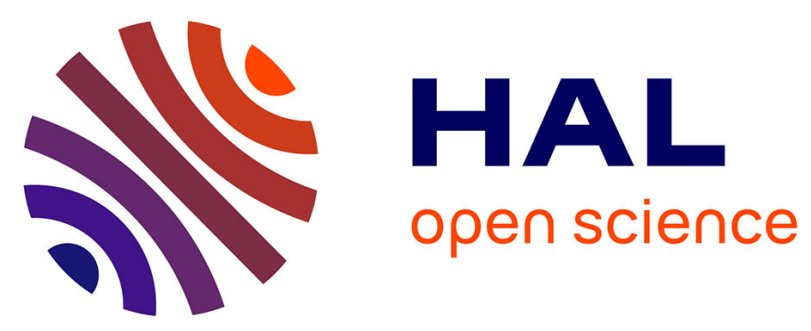

\title{
Restaurer la vision de patients aveugles avec des prothèses rétiniennes: Résultats cliniques et défis futurs
}

Amel Bendali, Henri Lorach, Milan Djilas, Olivier Marre, Ryad Bensoman, Lionel Rousseau, Gaelle Bazin Lissorgues, Emmanuel Scorsone, Philippe Bergonzo, José Garrido, et al.

\section{To cite this version:}

Amel Bendali, Henri Lorach, Milan Djilas, Olivier Marre, Ryad Bensoman, et al.. Restaurer la vision de patients aveugles avec des prothèses rétiniennes: Résultats cliniques et défis futurs. Biologie Aujourd'hui, 2013, 207 (2), pp.123-132. 10.1051/jbio/2013008 . cea-01791334

\section{HAL Id: cea-01791334 https://hal-cea.archives-ouvertes.fr/cea-01791334}

Submitted on 11 Feb 2019

HAL is a multi-disciplinary open access archive for the deposit and dissemination of scientific research documents, whether they are published or not. The documents may come from teaching and research institutions in France or abroad, or from public or private research centers.
L'archive ouverte pluridisciplinaire HAL, est destinée au dépôt et à la diffusion de documents scientifiques de niveau recherche, publiés ou non, émanant des établissements d'enseignement et de recherche français ou étrangers, des laboratoires publics ou privés. 


\title{
Restaurer la vision de patients aveugles avec des prothèses rétiniennes : Résultats cliniques et défis futurs
}

\author{
Amel Bendali ${ }^{1,2,3}$, Henri Lorach ${ }^{1,2,3}$, Milan Djilas ${ }^{1,2,3}$, Olivier Marre ${ }^{1,2,3}$, Ryad Bensoman ${ }^{1,2,3}$, \\ Lionel Rousseau ${ }^{4}$, Gaëlle Lissorgues ${ }^{4}$, Emmanuel Scorsone ${ }^{5}$, Philippe Bergonzo ${ }^{5}$, Jose A. Garrido ${ }^{6}$, \\ José Alain Sahel ${ }^{1,2,3,7,8,9}$ et Serge Picaud ${ }^{1,2,3,7}$ \\ 1 INSERM, U968, Institut de la Vision, 75012 Paris, France \\ 2 UPMC Université Paris 06, UMR S968, Institut de la Vision, 75012 Paris, France \\ 3 CNRS, UMR 7210, Institut de la Vision, 75012 Paris, France \\ 4 ESIEE-ESYCOM Université Paris Est, Cité Descartes, BP 99, 93162 Noisy Le Grand, France \\ 5 CEA, LIST, Diamond Sensor Laboratory, CEA/Saclay, 91191 Gif-sur-Yvette, France \\ 6 Walter Schottky Institut, Technische Universität München, 85748 Garching, Allemagne \\ 7 Fondation Ophtalmologique Adolphe de Rothschild, 75019 Paris, France \\ 8 Centre Hospitalier National d'Ophtalmologie des Quinze-Vingts, 75012 Paris, France \\ 9 Institute of Ophthalmology, University College of London, 11-43 Bath St, Greater London EC1V 9EL, Royaume Uni
}

Auteur correspondant : Serge Picaud, serge.picaud@inserm.fr

Reçu le 24 janvier 2013

Résumé - Les prothèses rétiniennes visent à restaurer une fonction visuelle utile chez des patients devenus aveugles par suite de la perte de leurs photorécepteurs. Les essais cliniques récents ont démontré la faisabilité de cette approche. Malgré un nombre limité d'électrodes (60) et donc a fortiori de pixels, certains patients sont en effet capables de lire des mots ou de reconnaître des objets très contrastés. Ces résultats soulèvent de nouveaux défis pour augmenter le nombre et la densité des électrodes afin d'améliorer l'acuité visuelle des patients pour autoriser des tâches complexes comme la locomotion autonome ou la reconnaissance des visages. Cette revue présente les résultats des essais cliniques sur les dispositifs les plus avancés puis les défis et solutions envisagées.

Mots clés : Neuroprothèses / Vision / Rétine / Cécité / Réhabilitation

Abstract - Restoring vision in blind patients following photoreceptor degeneration: clinical results and future challenges.

Retinal prostheses aim at restoring vision in patients blind from photoreceptor degeneration by electrically stimulating the residual retinal tissue. Currently, the most efficient implants are either inserted in the subretinal space or on the vitreal side of the retina (epi-retinal). Although the residual tissue can partly degenerate, it was shown that acute stimulation of residual neurones can induce visual percepts. Recently, a clinical trial with the epiretinal Argus2 device (60 electrodes) from the company $2^{\text {nd }}$ Sight enabled most patients to orient and find light targets, some even reading words. This device has received a CE mark. Surprisingly, when the subretinal implant from the company Retina Implant AG displaying many more electrodes (1500 electrodes) was evaluated in clinical trials, the patient visual performances were fairly similar. The restored visual performances of the patients demonstrate that blind patients can recover some visual function when their residual retina is properly stimulated. However, the resolution is not yet sufficient to perform complex tasks such as autonomous locomotion, face identification or text reading. Several challenges remain to generate an increase in pixel density corresponding to the increase in electrode number and 
density. These challenges include the stimulation modality, the tissue/implant interface design, the electrode materials, and the visual information encoder. This review will discuss these great challenges after introducing the major clinical results.

Key words: Neuroprostheses / vision / retina / blindness / vision recovery

\section{Introduction}

La cécité affecte 39 millions de personnes à travers le monde. Si la cataracte, principale cause de cécité dans le monde $(51 \%)$ peut être traitée, les autres causes résultent de pathologies rétiniennes souvent sans traitement ou dont les traitements ne sont malheureusement pas suffisamment performants. Ces pathologies rétiniennes sont le glaucome, la dégénérescence maculaire liée à l'âge (DMLA), la rétinopathie diabétique ou les dystrophies héréditaires comme la rétinopathie pigmentaire (RP). Dans ces maladies, la perte visuelle résulte soit de la dégénérescence des photorécepteurs, soit de celle des cellules ganglionnaires de la rétine qui communique l'information visuelle au cerveau. La perte des photorécepteurs intervient par exemple dans les RP et la DMLA, tandis que les cellules ganglionnaires de la rétine disparaissent dans la rétinopathie diabétique ou le glaucome. Pour certaines de ces maladies, il n'existe actuellement aucun traitement efficace pour prévenir la perte visuelle conduisant à la cécité. Les prothèses visuelles visent à restaurer une fonction visuelle chez les patients atteints de ces maladies et qui sont devenus aveugles. Il s'agit de stimuler électriquement les cellules du système visuel pour réintroduire des informations visuelles dans le circuit. Cependant, en fonction de la pathologie, et donc des cellules ayant dégénéré, il est possible d'intervenir à différents niveaux dans le système visuel. Lorsqu'il s'agit d'une perte des photorécepteurs, les prothèses rétiniennes visent à stimuler la rétine résiduelle alors que dans le cas d'une pathologie des cellules ganglionnaires de la rétine, il faudra activer les cellules du cortex visuel. Pour ce dernier type d'implants, des tests ont été réalisés dans les années 70-80 montrant une récupération fonctionnelle souvent transitoire avec des prothèses comprenant 100 électrodes (Dobelle et al., 1974; Dobelle, 2000). Le manque de persistance de la restauration visuelle a abouti à l'arrêt des essais cliniques dans l'attente d'une solution stable.

Pour les pathologies affectant les photorécepteurs (PR in figure 1), les prothèses rétiniennes ont été placées à différents niveaux. En effet, la perte des photorécepteurs laisse deux couches neuronales dans la rétine : 1) la couche interne dans laquelle se trouvent les cellules bipolaires (CB in figure 1) postsynaptiques aux photorécepteurs et 2) la couche des cellules ganglionnaires de la rétine (CGR in figure 1) dont les axones forment le nerf optique (NO) envoyant les informations visuelles au cerveau. Dans le fonctionnement normal de la rétine, ces deux couches neuronales opèrent un traitement du signal pour transformer les informations visuelles analogiques des photorécepteurs (leur potentiel de membrane est une fonction linéaire de l'intensité lumineuse) en des informations digitales où le code est contenu dans la fréquence des potentiels d'action des cellules ganglionnaires. Après la perte des photorécepteurs, une prothèse rétinienne peut être placée (1) dans l'espace sous-rétinien à la place d'origine des photorécepteurs (implant sous-rétinien); (2) à la surface côté vitré de la rétine à proximité des cellules ganglionnaires (implant épi-rétinien) ou voire (3) sous forme de gaine autour du nerf optique (figure 1). Si l'ensemble de ces implants peut produire une perception visuelle, les gaines autour du nerf optique n'ont pas permis de produire des images cohérentes (Veraart et al., 1998). Une approche transchoroïdale a également été proposée récemment (Morimoto et al., 2011). Cette revue présente uniquement les implants sous-rétiniens et épirétiniens dans les essais cliniques et les défis futurs de ces prothèses rétiniennes.

\section{Les essais cliniques}

Les prothèses neuronales ont déjà trouvé des applications pour compenser différentes pertes neuronales intervenant dans plusieurs pathologies neurodégénératives. Les applications cliniques les plus courantes portent sur le traitement de la maladie de Parkinson par des électrodes profondes dans le noyau sous-thalamique et sur le traitement du déficit auditif. En effet, pour cet autre déficit sensoriel, les implants cochléaires permettent maintenant à des patients sourds de suivre une conversation téléphonique. Ces succès ont stimulé la recherche sur les prothèses rétiniennes. L'idée de redonner une perception visuelle à des aveugles est relativement ancienne puisqu'il faut remonter en 1755 à Charles Leroy qui a le premier appliqué une décharge électrique sur la surface oculaire d'un patient aveugle du fait d'une cataracte. Le patient avait alors perçu des clignotements vifs de lumière ou phosphènes.

\section{Réactivation des cellules rétiniennes}

Dans le cas de dégénérescence des photorécepteurs, la première question posée concerne la possibilité de 

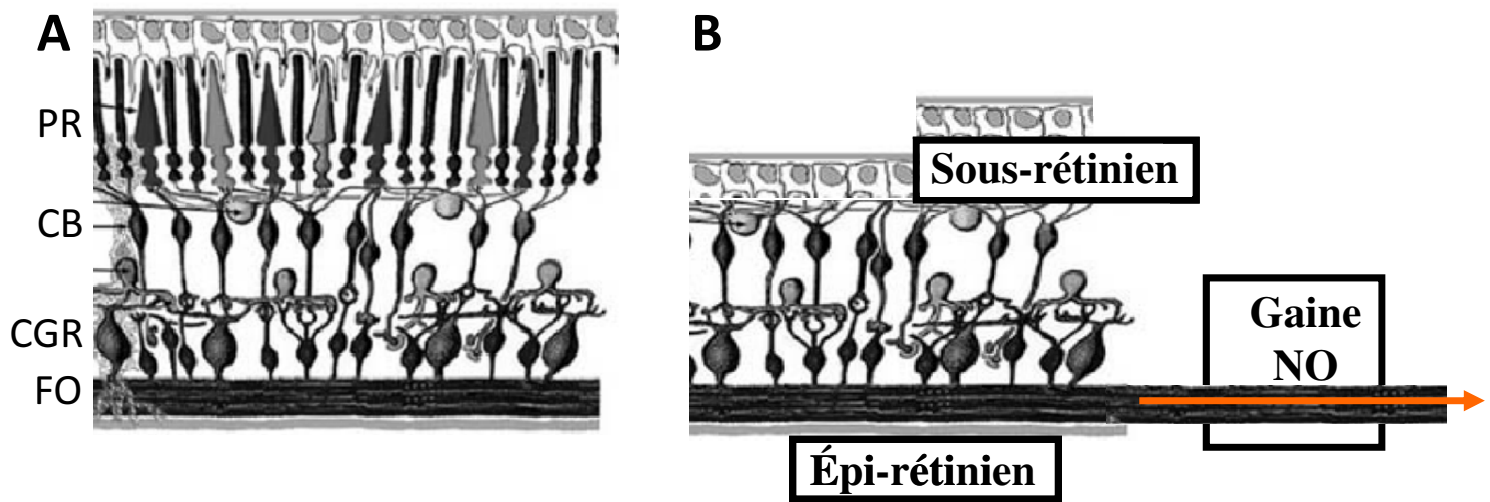

Fig. 1. Concept des prothèses rétiniennes. (A) Schéma d'une rétine présentant les différentes couches neuronales : la couche des photorécepteurs (PR), la couche nucléaire interne contenant les cellules bipolaires (CB), la couche des cellules ganglionnaires rétiniennes (CGR) et la couche des fibres optiques ou axones (FO) se dirigeant vers le nerf optique (NO). (B) Schéma présentant la position des implants sous-rétiniens entre rétine résiduelle et epithélium pigmentaire, des implants epi-rétiniens sur la rétine côté vitré et des gaines d'électrodes placées autour du NO. (Adapté à partir de Webvision).

réactiver le tissu résiduel d'autant que ce dernier est lui aussi sujet à une dégénérescence secondaire des neurones. Il a ainsi été rapporté que les patients à un stade avancé de rétinopathies pigmentaires ne conservent qu'un tiers à un quart de leurs cellules ganglionnaires rétiniennes (Humayun et al., 1999). Un remodelage du tissu rétinien et une perte des cellules ganglionnaires ont également été rapportés sur les modèles animaux de la maladie (Wang et al., 2000 ; Marc et al., 2003; Marc et al., 2007). Différentes causes de cette dégénérescence ont été proposées dans la littérature (Villegas-Perez et al., 1998; Froger et al., 2012). La question posée était donc de déterminer si ces cellules dans un état de dégénérescence seraient encore en mesure de transmettre un signal au cerveau et s'il serait toujours interprété comme une perception visuelle. Les premiers tests cliniques ont donc consisté en des études en conditions aiguës au cours desquelles une électrode ou une matrice d'électrodes était appliquée contre la rétine du côté vitréen. Ces expériences ont montré que les patients percevaient des phosphènes pouvant prendre différentes formes suivant la stimulation (Humayun et al., 1996; Rizzo et al., 2003b, 2003a). Ces résultats ont ainsi permis de démontrer la possibilité de réactiver électriquement la rétine de patients aveugles.

\section{Implants épi-rétiniens}

Après avoir démontré la capacité de la rétine résiduelle à communiquer une information visuelle, les essais cliniques ont ensuite porté sur des prothèses rétiniennes comportant une matrice d'électrodes implantée en chronique. Les prothèses épi-rétiniennes sont composées des éléments suivants : (1) une caméra située sur des lunettes pour acquérir les images de l'environnement visuel; (2) un microprocesseur qui transforme les images en matrices de stimulations électriques; (3) un émetteur qui transmet l'information par radiofréquence vers le dispositif implanté; (4) sur le dispositif implanté, le receveur de radiofréquence (interaction bobine-bobine) est relié à un ASIC (puce électronique) qui permet de transformer les informations de radiofréquence en courants électriques sur les différentes voies de stimulations; (5) sortant du receveur, l'implant proprement dit contient les différentes pistes électriques conduisant aux différentes électrodes, et l'extrémité de cet implant contenant les électrodes est placée au contact de la rétine et sa fixation est assurée par un tack ou clou traversant la choroïde. La société 2nd Sight a dans un premier temps produit un implant avec 16 électrodes qui a permis de mettre en évidence la possibilité pour les patients d'identifier une cible lumineuse voire de reconnaître des objets blancs sur fond noir avec une acuité visuelle retrouvée de 20/3240 (Humayun et al., 2003; Caspi et al., 2009). La figure 2 illustre le dispositif Argus2, disposant cette fois de 60 électrodes. Cette nouvelle génération d'implants a fait l'objet d'une étude multicentrique internationale incluant 30 patients entre 2007 et 2009, dont quatre furent opérés au Centre Hospitalier National d'Ophtalmologie des XV-XX à Paris dans le service dirigé par le Pr Sahel, co-auteur de cette publication. Avec ce dispositif, la majorité des patients pouvait réaliser des tâches visuelles complexes telles que la localisation d'objets (96\% des sujets), la discrimination du mouvement $(57 \%)$, et la discrimination de réseaux orientés $(23 \%)$ (Humayun et al., 2012). La 

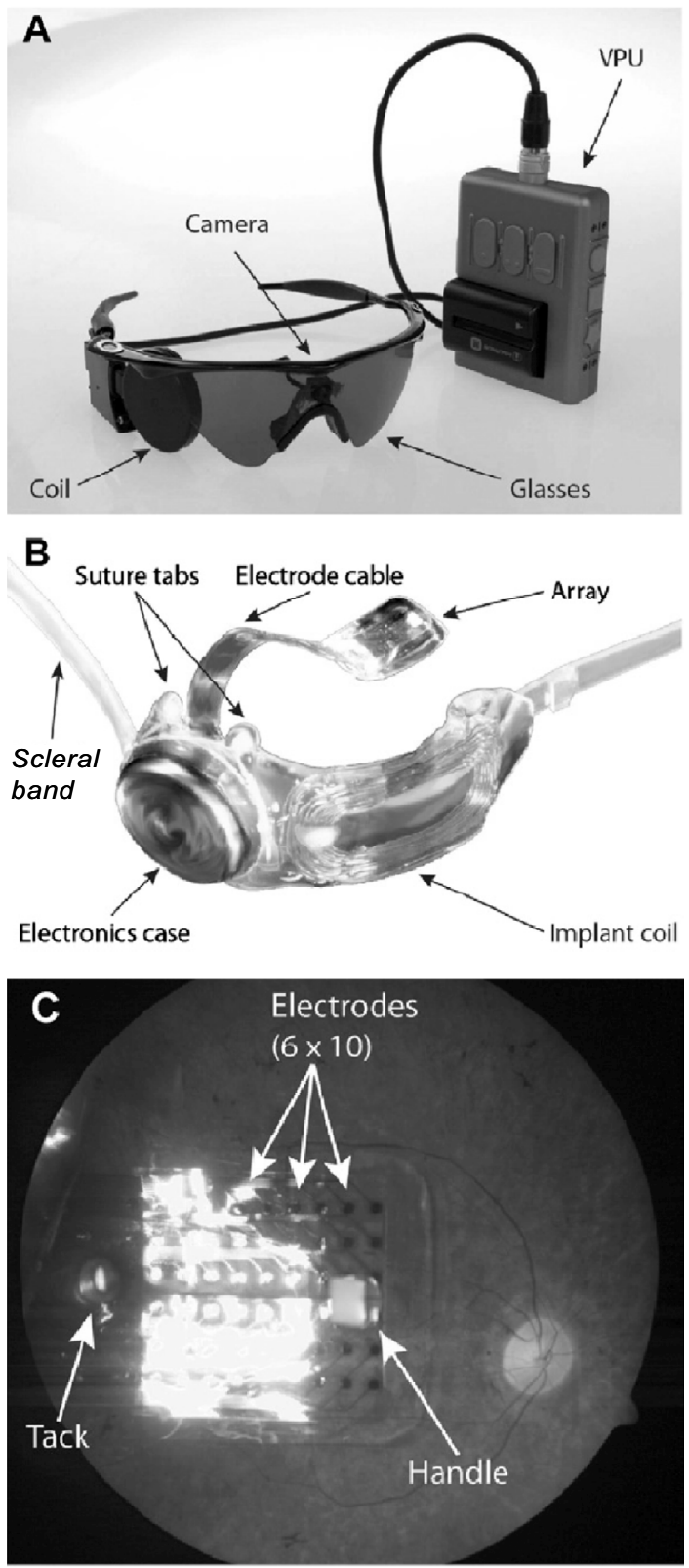

Fig. 2. Première prothèse rétinienne commercialisée : L'Argus2 de l'entreprise $2^{\text {nd }}$ Sight Medical products. (A) Partie externe du dispositif contenant le microprocesseur (VPU) et la paire de lunettes sur laquelle se trouve la caméra et la bobine de transmission radiofréquence ( Coil). (B) Partie implantable du dispositif contenant le boîtier électronique (Electronics case) attaché autour de l'œil par un bandeau (Scleral band) sur lequel on retrouve la bobine de réception en radiofréquence (Implant coil), l'implant pénétrant dans l'œil se constitute du cable (Electrode cable) le reliant au boîtier et la matrice d'électrodes (Array). (C) Image du fond d'œil présentant la matrice d'électrodes, son clou de fixation (Tack) et la partie permettant de manipuler la prothèse lors de la chirurgie (Handle) (D'après Humayun et al., 2012). meilleure acuité visuelle mesurée était de 20/1260, encore six fois inférieure à la limite de la cécité légale (20/200). Deux des patients implantés ont également pu effectuer des tâches de lecture avec un taux pouvant aller jusqu'à 10 mots par minute. Ces succès ont permis à ce dispositif médical d'obtenir le marquage CE pour sa commercialisation en Europe au prix de vente de $80.000 €$ et déjà l'accord pour son remboursement en Allemagne par l'agence sanitaire de ce pays. D'autres groupes ont également réalisé des essais cliniques confirmant la possibilité de restaurer une certaine perception visuelle avec un implant épi-rétinien (Zeitz et al., 2009; Klauke et al., 2011).

Le dispositif Argus2 stimule le tissu par des courants dont l'amplitude est une fonction linéaire de l'intensité du pixel considéré à une fréquence d'environ $20 \mathrm{~Hz}$ pour une durée d'impulsion de $100 \mu \mathrm{s}$ à $1 \mathrm{~ms}$ avec une stimulation bi-phasique, cathodique puis anodique. Cette stratégie fournit donc exactement les mêmes informations à tous les types de cellules ganglionnaires sans tenir en compte de leur diversité (Roska \& Werblin, 2001). Les cellules s'activant à l'allumage d'une lumière, cellules $O N$, recevront donc la même stimulation que celles qui s'activent à l'extinction de la phase lumineuse, cellules OFF. Outre la stimulation identique de toutes les cellules ganglionnaires de la rétine, l'approche épi-rétinienne induit également une stimulation des fibres passant à la surface de la rétine en direction du nerf optique (Rizzo et al., 2003b; Wilms \& Eckhorn, 2005). Cette stimulation des fibres revient à une stimulation de cellules ganglionnaires plus périphériques et donc à une perte de la rétinotopie ou perte de la projection exacte du champ visuel sur la rétine. Ces différentes difficultés liées aux stimulations épi-rétiniennes pourraient expliquer la variabilité dans les performances des patients implantés avec l'Argus2, seulement sept des 30 patients étant capables de réaliser de façon fiable les tâches d'acuité visuelle (Humayun et al., 2012).

\section{Implants sous-rétiniens}

L'approche sous-rétinienne pourrait permettre de limiter ou de supprimer ces différentes difficultés. L'entreprise Optobionics fut la première à évaluer de tels implants qui reposaient sur des photodiodes solaires directement insérées dans l'implant pour produire un courant de stimulation. Le dispositif devient donc très simple puisque la puce électronique sousrétinienne comprend les photodiodes et les électrodes. Aucun élément externe n'est nécessaire, il faut simplement décoller la rétine résiduelle pour insérer dans l'espace sous-rétinien le dispositif circulaire de deux millimètres de diamètre. Malheureusement, les photodiodes ne permettaient pas une conversion de photons 
en nombre suffisant pour entraîner l'activation du tissu rétinien. Seul un effet trophique à distance a pu être observé avec ce dispositif (Chow et al., 2004). Pour résoudre ce problème technique, le Pr Zrenner et la société Retina Implant AG ont introduit un circuit amplificateur des signaux issus des photodiodes (Zrenner, 2002). La puce reproduit donc exactement le fonctionnement d'un photorécepteur, réception des photons, amplification de ce signal, activation des neurones bipolaires. Cependant, la présence du circuit amplificateur impose l'addition d'une batterie qui est également placée dans l'espace sous-rétinien mais en grande périphérie. Ce système rend par conséquent l'opération chirurgicale beaucoup plus complexe puisqu'elle oblige à insérer le dispositif dans l'espace sousrétinien à partir de la périphérie jusqu'en position centrale. Dans un premier temps, le fonctionnement imparfait du dispositif a été compensé par la présence d'une matrice à 16 électrodes sous-rétiniennes reliées à l'extérieur par une liaison filaire ce qui avait permis de démontrer l'obtention d'une perception visuelle sous l'effet d'une stimulation sous-rétinienne (Besch et al., 2008; Wilke et al., 2011). Dans une seconde phase, le fonctionnement de la puce qui contient 1500 électrodes a pu être testé de manière autonome. Avec ce dispositif, les patients perçoivent des objets lumineux, discriminent des mires simples et peuvent dans certains cas lire les lettres. Zrenner et al. (2011) ont rapporté une acuité visuelle de 20/1000 chez ces patients. Ces résultats peuvent paraître décevants puisque les performances visuelles sont du même ordre que celles des patients implantés avec l'Argus2 alors que les études psychophysiques estimaient que des implants à 600 pixels devraient permettre la reconnaissance des visages, la lecture de textes complexes et la locomotion autonome (Cha et al., 1992a, 1992b; Sommerhalder et al., 2003, 2004; Perez Fornos et al., 2008). Cette incohérence avec la théorie pourrait s'expliquer par une diaphonie entre les électrodes voisines séparées de $70 \mu \mathrm{m}$ qui partagent une électrode de masse très lointaine. L'ensemble de ces résultats a apporté la preuve qu'un patient aveugle peut retrouver une vision utile avec une prothèse rétinienne. Il faut maintenant relever différents défis pour augmenter la résolution des électrodes pour améliorer l'acuité visuelle des patients et pour simplifier les dispositifs rendant ainsi la chirurgie plus aisée.

\section{Les défis du futur}

\section{Le mode de stimulation}

Les travaux du Pr Zrenner et de l'entreprise Retina Implant AG ont démontré que le fait d'augmenter le nombre et la densité des électrodes ne repose pas sur la seule prouesse des électroniciens mais qu'il faut sûrement repenser le design de la puce électronique pour que chaque électrode produise une stimulation rétinienne indépendante de sa voisine. Le mode de stimulation, monopolaire, bipolaire ou par rapport à un plan de masse peut produire une focalisation importante des courants (Joucla \& Yvert, 2009). C'est justement l'approche choisie par le groupe du Pr Palanker qui réintroduit le concept de photodiode complètement autonome avec une électrode en référence d'un plan de masse local (Mathieson et al., 2012; Wang et al., 2012). Dans ce dispositif, chaque électrode est reliée à trois photodiodes en série fournissant suffisamment de courant pour évoquer une activation du tissu rétinien. Les photodiodes sont sensibles à la lumière infrarouge qui devra donc être projetée par un projecteur monté dans une paire de lunettes et recevant les informations visuelles d'une caméra.

\section{Le design de l'interface}

Le groupe du Pr Palanker a également soulevé la question du design de l'interface implant/tissu. En effet, de chaque côté de la rétine résiduelle, on retrouve une couche cellulaire gliale qui agit comme une couche isolante à la surface de la rétine. Par conséquent, le courant de stimulation aura plus tendance à circuler dans l'espace séparant l'implant du tissu qu'à pénétrer dans le tissu. C'est pourquoi le Pr Palanker a proposé de réaliser des implants tridimensionnels permettant de placer les neurones entre les deux électrodes de polarité opposée (Palanker et al., 2004). Les structures proposées sont plus particulièrement des piliers pénétrant progressivement dans le tissu pour s'approcher des neurones (Butterwick et al., 2009). Plus récemment, nous avons repris cette idée pour définir une structure optimale de tels implants tridimensionnels. Cependant, au lieu de piliers qui empêcheraient tout retrait du dispositif en cas de problème, nous avons proposé de réaliser des puits avec une électrode au fond et un plan de masse à la surface de l'implant. Une modélisation a permis de valider cette approche qui aboutirait à la stimulation de colonnes de cellules rétiniennes (figure 3A) (Djilas et al., 2011). Cependant, cette stratégie repose sur l'hypothèse selon laquelle la rétine résiduelle va se mouler dans les puits creusés dans l'implant. Par conséquent, nous avons examiné si la rétine résiduelle possède cette plasticité en introduisant des prototypes d'implants 3D sous la rétine de rats aveugles (figure 3B, 3C). Les résultats montrent que la rétine se moule dans les puits où l'on retrouve des cellules bipolaires (figure 3D) qui pourraient donc être activées sélectivement indépendamment des cellules bipolaires isolées dans les puits voisins (Djilas et al., 2011). Le design tridimensionnel des implants 

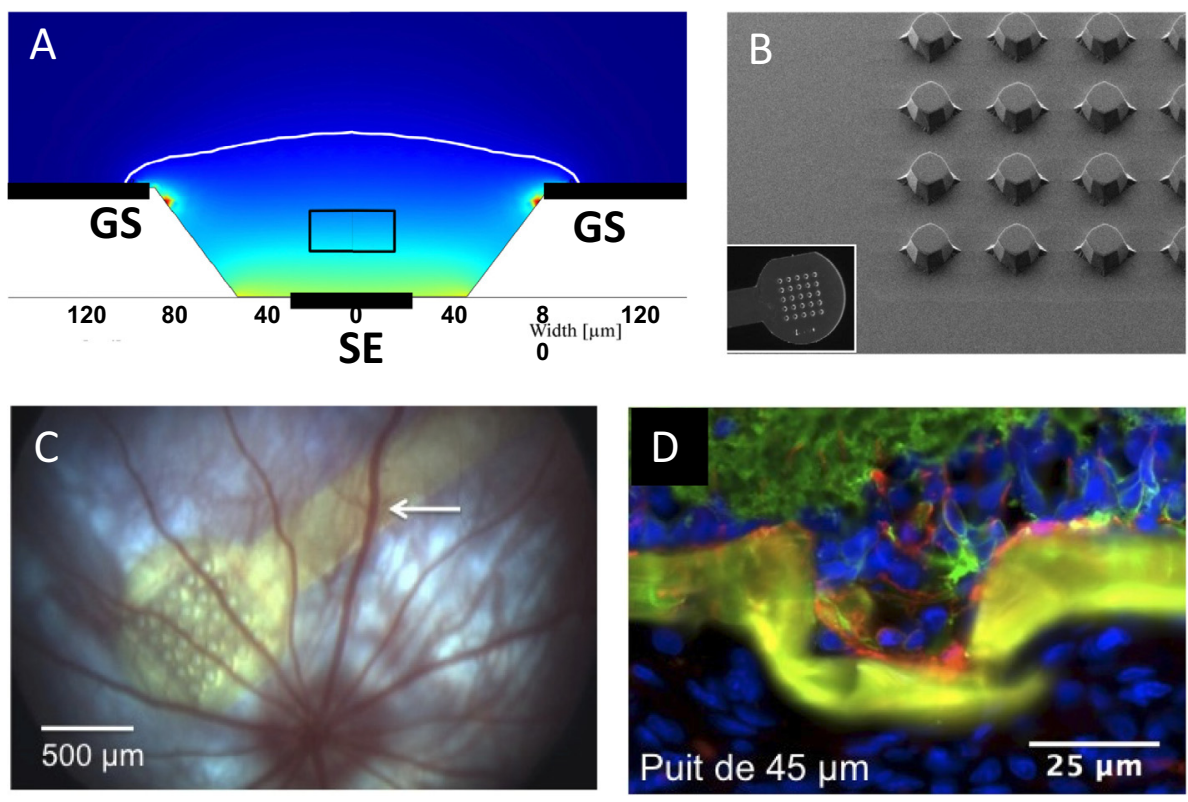

Fig. 3. Focalisation des stimulations par des interfaces tridimensionnelles. (A) Modélisation des intensités de courants dans un tissu au contact d'un implant tridimensionel avec une électrode de stimulation (SE) au fond du puits et une grille de masse à la surface de l'implant et sur les parois du puits (GS). Le code couleur indique un confinement des courants dans le puits et notamment dans la zone cible symbolisée par un rectangle. (B) Moule ayant servi pour la production d'un prototype d'implant tridimensionnel en insert. (C) Fond d'œil d'un rat aveugle montrant l'implant tridimensionnel sous la rétine et donc sous les vaisseaux rétiniens (flèche). (D) Coupe histologique de la rétine du rat aveugle montrant les neurones dans le puits. (Adapté d'après Djilas et al., 2011).

sous-rétiniens pourrait donc permettre de considérer chaque électrode comme un pixel indépendant.

\section{Les matériaux}

Pour la stimulation électrique d'une structure neuronale, deux types de courants peuvent être générés par les électrodes : (1) les courants faradiques qui impliquent des réactions chimiques d'oxydation/réduction; (2) les courants capacitifs qui résultent seulement de l'accumulation de charges. Dans le cas des prothèses neuronales, les stimulations capacitives sont préférées car elles limitent les modifications de $\mathrm{pH}$ et du tissu à la surface de l'électrode (Cogan, 2008). Dans le contexte des implants visuels, la nécessité d'utiliser des petites électrodes introduit un risque supplémentaire de dégradation des tissus et des électrodes par l'injection de densités de charges élevées. Trouver des matériaux à fortes limites d'injection de charges est donc essentiel. Les matériaux classiquement utilisés sont le platine (limite d'injection $\left.0,35 \mathrm{mC} / \mathrm{cm}^{2}\right)$, le nitrure de titane $\left(1 \mathrm{mC} / \mathrm{cm}^{2}\right)$ et l'oxyde d'iridium $\left(4 \mathrm{mC} / \mathrm{cm}^{2}\right)$. L'étude de ces matériaux implique des évaluations de leur biocompatibilité sur des cellules rétiniennes comme par exemple pour le nitrure de titane (Guenther et al., 1999). D'autres matériaux sont actuellement à l'étude comme le diamant qui peut devenir un semi-conducteur dès lors qu'il est dopé par des atomes de bore. Nous avons ainsi caractérisé de telles électrodes de diamant (Kiran et al., 2012) et évalué la biocompatibilité de ce matériau pour les cellules rétiniennes (Bergonzo et al., 2012). D'autres ont également estimé l'intérêt du diamant pour la stimulation électrique de cellules rétiniennes (Hadjinicolaou et al., 2012). En parallèle, nous avons également analysé la biocompatibilité du graphène pour les cellules rétiniennes (Bendali et al., 2013), ce matériau présentant également des qualités électroniques très intéressantes. La figure 4 illustre par exemple la survie de cellules ganglionnaires rétiniennes adultes sur du graphène, qu'il soit nu ou recouvert de protéines (coating). Ces nouveaux matériaux pourraient donc apporter des perspectives inédites liées à leur structure carbonée, proche de la matière vivante.

\section{Codage de l'information visuelle}

Actuellement, le codage des informations visuelles est relativement simple, l'intensité lumineuse étant transformée en une amplitude de stimulation. En réalité, nombreux pensent qu'en dépit d'un codage précis de l'information visuelle, le cortex devrait être en mesure de réapprendre à voir et ainsi d'extraire les informations pertinentes. Cette plasticité du 

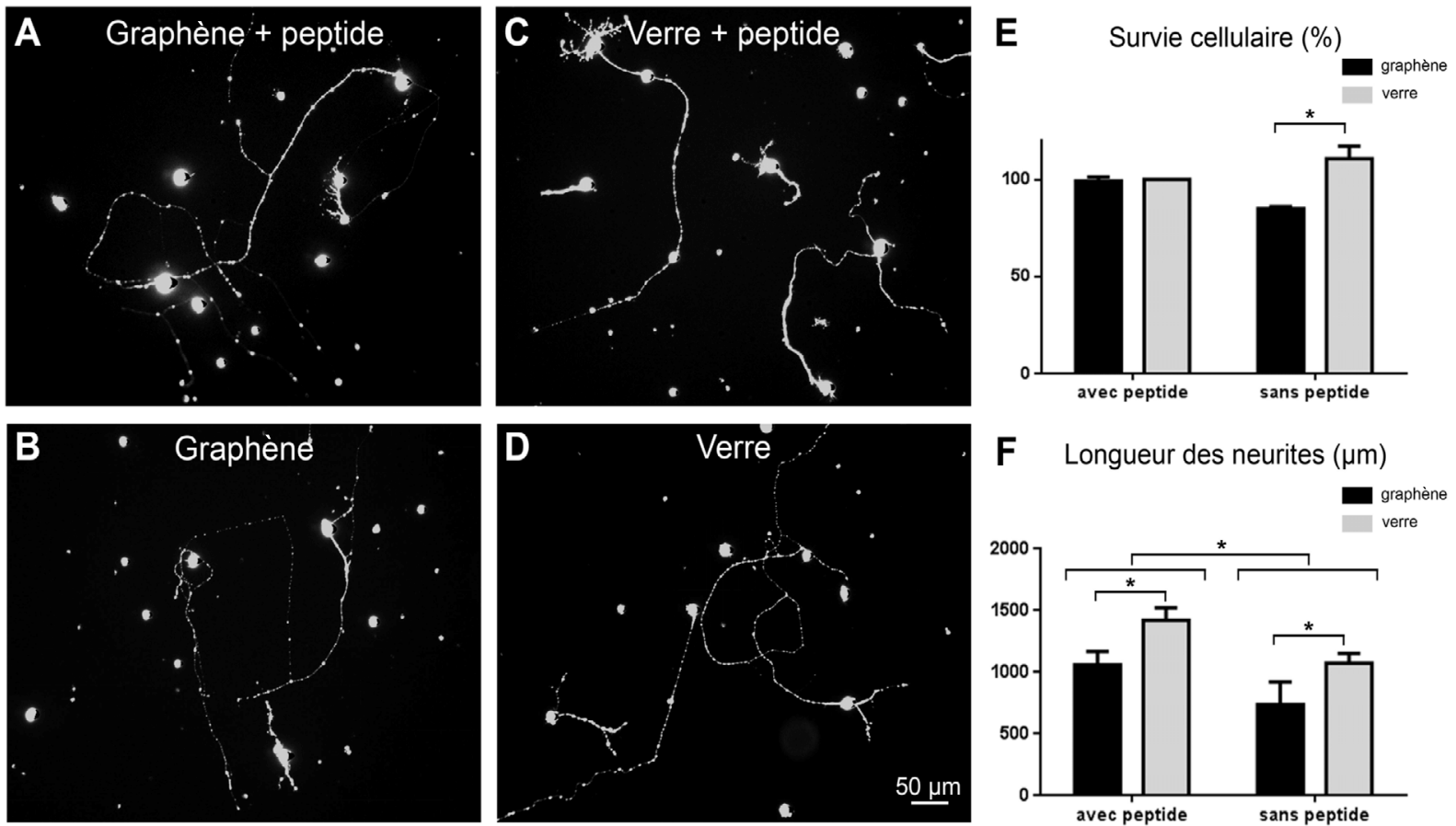

Fig. 4. Survie de cellules ganglionnaires rétiniennes adultes sur du graphène ou sur verre soit nu soit recouvert de protéines (coating : poly-D-lysine et laminine). (A)-(D) : Visualisation des cellules viables par un marquage calcéine en fluorescence sur les différents supports. (E)-(F) : Quantification des cellules viables et de leur développement neuritique. (Moyennes \pm s.e.m. $n=4$ ). ${ }^{*} p<0.05$. L'échelle représente $50 \mu \mathrm{m}$. (Adapté d'après Bendali et al., 2013).

système visuel des patients aveugles repose sur sa réorganisation fonctionnelle après apparition de la cécité (Amedi et al., 2010; Striem-Amit et al., 2012). Chez les patients aveugles, en effet, le cortex visuel peut être recruté par d'autres modalités sensorielles introduisant une plasticité intermodale, le cortex visuel contribuant à la lecture tactile du braille (Cohen et al., 1997). Cependant, plus le signal induit sera proche de la réponse physiologique, plus on peut espérer que le patient sera en mesure d'interpréter le nouveau langage visuel proposé. Cette difficulté pourrait d'ailleurs expliquer l'absence de compréhension des signaux chez certains patients.

Dans ce contexte, l'un des grands défis des prothèses visuelles est de développer des algorithmes de traitement de l'information visuelle fonctionnant en temps réel. Ces traitements doivent s'appliquer pour tous les types cellulaires dans la mesure où la stimulation peut intervenir à différents niveaux dans la rétine; or personne ne sait actuellement si des types cellulaires spécifiques sont plus sujets à la dégénérescence que d'autres dans la rétine interne. Différents modèles ont tenté de reproduire le fonctionnement des cellules ganglionnaires (Victor, 1987, 1988). Si des modèles plus récents reproduisent avec un certain succès les propriétés des cellules ganglionnaires, ils ne peuvent pas encore prédire de manière satisfaisante les réponses à des stimuli naturels complexes (Gollisch \& Meister, 2010). Par conséquent, il devient difficile de produire un encodeur d'images naturelles pour les prothèses rétiniennes comme certains le proposent (Nirenberg \& Pandarinath, 2012). Récemment, nous avons abordé cette question par une approche novatrice reposant sur une nouvelle caméra dite asynchrone dont la fonction mime celle des photorécepteurs et de la rétine (Lichtsteiner et al., 2008). L'avantage de ces caméras est l'absence d'horloges qui contraignent le traitement du signal par l'acquisition à intervalles de temps réguliers comme dans les caméras classiques (une image toutes les $30 \mathrm{~ms}$ ). Les pixels de cette caméra fonctionnent de manière totalement indépendante les uns des autres, signalant individuellement à la caméra par un événement soit positif soit négatif, le passage au seuil d'intensité lumineuse suivant dans les deux sens, positif et négatif. Utilisant ce type d'information, nous avons pu reconstruire les réponses physiologiques des cellules ganglionnaires préalablement décrites par Roska et Werblin (Roska \& Werblin, 2001). Dans notre cas, nous arrivons à une précision à la milliseconde des réponses cellulaires, ce qui permet d'extrapoler les réponses à des stimulations complexes (Lorach et al., 2012). La figure 5 illustre par exemple 


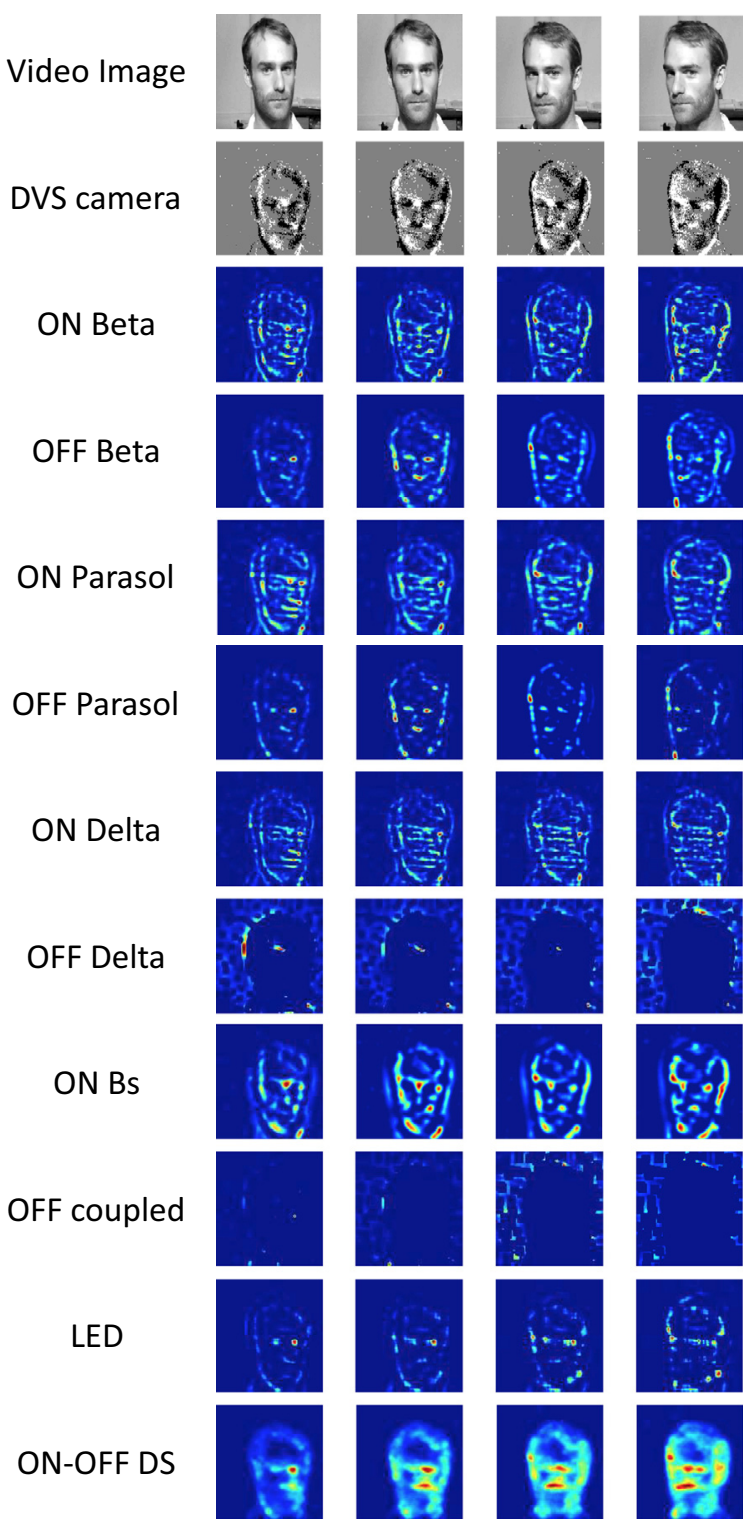

Fig. 5. Modélisation des informations visuelles envoyées au cerveau par différentes populations de cellules ganglionnaires rétiniennes. La modélisation est réalisée à partir des évènements positifs et négatifs (points blancs et noirs) générés par la caméra asynchrone de type DVS. L'intensité de la réponse d'une cellule est représentée par un code couleur au niveau du pixel correspondant (voir Lorach et al., 2012).

les images visuelles envoyées par la rétine au cerveau. Cette nouvelle stratégie de codage de l'information visuelle devrait donc nous permettre de procurer aux patients une information visuelle proche de celle que les cellules génèrent normalement et donc plus facilement interprétable.

\section{Conclusions}

La restauration visuelle par des prothèses rétiniennes devient une approche réaliste puisque certains patients ont montré des performances visuelles remarquables avec des implants contenant seulement 60 électrodes. Nous sommes sûrement au début de cette aventure qui nécessitera différentes améliorations des systèmes existants. Cependant, comme pour les implants cochléaires qui ont nécessité 20 ans de développement entre la reconnaissance d'un mot et la compréhension d'une phrase, nous pouvons espérer que des patients puissent d'ici quelques années retrouver une certaine autonomie dans leur déplacement, la reconnaissance visuelle de leurs proches ainsi que des tâches usuelles comme la lecture. L'innovation doit être source de cet espoir pour de nombreux patients d'autant que les développements sur le système visuel pourraient ensuite ouvrir la voie pour d'autres prothèses neuronales dans le traitement d'autres pathologies.

Remerciements. $\mathrm{AB}$ et $\mathrm{HL}$ ont reçu une bourse de doctorat, respectivement de l'UPMC et de l'École Polytechnique. Ce projet est soutenu par l'Inserm, l'Université Pierre et Marie Curie (Paris VI), la Fondation Ophtalmologique A. de Rothschild (Paris), l'Agence Nationale pour la Recherche (ANR RETINE, ANR MEDINAS), le programme national français « Investissement d'Avenir » [LIFESENSES : ANR-10-LABX-65], ITMO Technologie Pour la Santé, la Fédération des Aveugles de France, IRRP, la ville de Paris, le Conseil régional d'Ile-de-France, la commission Européenne (projets DREAMS et NEUROCARE) et la Fondation pour la Recherche Médicale.

\section{Références}

Amedi A., Raz N., Azulay H., Malach R., Zohary E., Cortical activity during tactile exploration of objects in blind and sighted humans. Restor Neurol Neurosci, 2010, 28, 143-156.

Bendali A., Hess L.H., Seifert M., Forster V., Stephan A.F., Garrido J.A., Picaud S., Purified neurons can survive on peptide-free graphene layers. Adv Healthcare Mater, 2013, 2, 929-933.

Bergonzo P., Bongrain A., Scorsone E., Bendali A., Rousseau L., Lissorgues G., Mailley P., Li Y., Kauffmann T., Goy F., Yvert B., Sahel J.A., Picaud S., 3D shaped mechanically flexible diamond microelectrode arrays for eye implant applications: The MEDINAS project. IRBM, 2012, 32, 91-94.

Besch D., Sachs H., Szurman P., Gulicher D., Wilke R., Reinert S., Zrenner E., Bartz-Schmidt K.U., Gekeler F., Extraocular surgery for implantation of an active subretinal visual prosthesis with external connections: feasibility and outcome in seven patients. Brit $J$ Ophthalmol, 2008, 92,1361-1368. 
Butterwick A., Huie P., Jones B.W., Marc R., Marmor M., Palanker D., Effect of shape and coating of a subretinal prosthesis on its integration with the retina. Exp Eye Res, 2009, 88, 22-29.

Caspi A., Dorn J.D., McClure K.H., Humayun M.S., Greenberg R.J., McMahon M.J., Feasibility study of a retinal prosthesis: spatial vision with a 16-electrode implant. Arch Ophthalmol, 2009, 127, 398-401.

Cha K., Horch K.W., Normann R.A., Mobility performance with a pixelized vision system. Vision Res, 1992a, 32, 1367-1372.

Cha K., Horch K.W., Normann R.A., Boman D.K., Reading speed with a pixelized vision system. J Opt Soc Am A, 1992b, 9, 673-677.

Chow A.Y., Chow V.Y., Packo K.H., Pollack J.S., Peyman G.A., Schuchard R., The artificial silicon retina microchip for the treatment of vision loss from retinitis pigmentosa. Arch Ophthalmol, 2004, 122, 460-469.

Cogan S.F., Neural stimulation and recording electrodes. Ann Rev Biomedl Eng, 2008, 10, 275-309.

Cohen L.G., Celnik P., Pascual-Leone A., Corwell B., Falz L., Dambrosia J., Honda M., Sadato N., Gerloff C., Catala M.D., Hallett M., Functional relevance of crossmodal plasticity in blind humans. Nature, 1997, 389, 180-183.

Djilas M., Oles C., Lorach H., Bendali A., Degardin J., Dubus E., Lissorgues-Bazin G., Rousseau L., Benosman R., Ieng S.H., Joucla S., Yvert B., Bergonzo P., Sahel J., Picaud S., Three-dimensional electrode arrays for retinal prostheses: modeling, geometry optimization and experimental validation. J Neural Eng, 2011, 8, 046020.

Dobelle W.H., Artificial vision for the blind by connecting a television camera to the visual cortex. Asaio J, 2000, 46, 3-9.

Dobelle W.H., Mladejovsky M.G., Girvin J.P., Artifical vision for the blind: electrical stimulation of visual cortex offers hope for a functional prosthesis. Science, 1974, 183, 440-444.

Froger N., Cadetti L., Lorach H., Martins J., Bemelmans A.P., Dubus E., Degardin J., Pain D., Forster V., Chicaud L., Ivkovic I., Simonutti M., Fouquet S., Jammoul F., Leveillard T., Benosman R., Sahel J.A., Picaud S., Taurine provides neuroprotection against retinal ganglion cell degeneration. PloS One, 2012, 7, e42017.

Gollisch T., Meister M., Eye smarter than scientists believed: neural computations in circuits of the retina. Neuron, 2010, 65, 150-164.

Guenther E., Troger B., Schlosshauer B., Zrenner E., Longterm survival of retinal cell cultures on retinal implant materials. Vision Res, 1999, 39, 3988-3994.

Hadjinicolaou A.E., Leung R.J., Garrett, D.J. Ganesan K., Fox K., Nayagam D., Shivdasani M.N., Meffin H., Ibbotson M.R., Prawer S., O'Brien B.J., Electrical stimulation of retinal ganglion cells with diamond and the development of an all diamond retinal prosthesis. Biomaterials, 2012, 33, 5812-5820.
Humayun M.S., de Juan Jr. E., Dagnelie G., Greenberg R.J., Propst R.H., Phillips D.H., Visual perception elicited by electrical stimulation of retina in blind humans. Arch Ophthalmol, 1996, 114, 40-46.

Humayun M.S., Prince M., de Juan Jr. E., Barron Y., Moskowitz M., Klock I.B., Milam A.H., Morphometric analysis of the extramacular retina from post-mortem eyes with retinitis pigmentosa. Invest Ophtalmol Vis Sci, 1999, 40, 143-148.

Humayun M.S., Weiland J.D., Fujii G.Y., Greenberg R., Williamson R., Little J., Mech B., Cimmarusti V., Van Boemel G., Dagnelie G., de Juan E., Visual perception in a blind subject with a chronic microelectronic retinal prosthesis. Vision Res, 2003, 43, 2573-2581.

Humayun M.S., Dorn J.D., da Cruz L., Dagnelie G., Sahel J.A., Stanga P.E., Cideciyan A.V., Duncan J.L., Eliott D., Filley E., Ho A.C., Santos A., Safran A.B., Arditi A., Del Priore L.V., Greenberg R.J., Interim results from the international trial of Second Sight's visual prosthesis. Ophthalmology, 2012, 119, 779-788.

Joucla S., Yvert B., Improved focalization of electrical microstimulation using microelectrode arrays: a modeling study. PloS One, 2009, 4, e4828.

Kiran R., Rousseau L., Lissorgues G., Scorsone E., Bongrain A., Yvert B., Picaud S., Mailley P., Bergonzo P., Multichannel boron doped nanocrystalline diamond ultramicroelectrode arrays: Design, fabrication and characterization. Sensors, 2012, 12, 7669-7681.

Klauke S., Goertz M., Rein S., Hoehl D., Thomas U., Eckhorn R., Bremmer F., Wachtler T., Stimulation with a wireless intraocular epiretinal implant elicits visual percepts in blind humans. Invest Ophthalmol Vis Sci, 2011, 52, 449-455.

Lichtsteiner P., Posch C., Delbruck T., A 128 times, 128 $120 \mathrm{db} 15$ us latency asynchronous temporal contrast vision sensor. Solid-State Circuits, IEEE Journal, 2008, 43,566-576.

Lorach H., Benosman R., Marre O., Ieng S.H., Sahel J.A., Picaud S., Artificial retina: The multichannel processing of the mammalian retina achieved with a neuromorphic asynchronous light acquisition device. J Neural Eng, 2012, 9, 066004.

Marc R.E., Jones B.W., Watt C.B., Strettoi E., Neural remodeling in retinal degeneration. Prog Retin Eye Res, 2003, 22, 607-655.

Marc R.E., Jones B.W., Anderson J.R., Kinard K., Marshak D.W, Wilson J.H., Wensel T., Lucas R.J., Neural reprogramming in retinal degeneration. Invest Ophthalmol Vis Sci, 2007, 48, 3364-3371.

Mathieson K., Loudin J., Goetz G., Huie P., Wang L., Kamins T., Smith R., Harris J.S., Sher A., Palanker D., Photovoltaic retinal prosthesis with high pixel density. Nat Photonics, 2012, 6, 391-397.

Morimoto T., Kamei M., Nishida K., Sakaguchi H., Kanda H., Ikuno Y., Kishima H., Maruo T., Konoma K., Ozawa M., Nishida K., Fujikado T., Chronic implantation of newly developed suprachoroidal-transretinal stimulation prosthesis in dogs. Invest Ophtalmol Vis Sci, 2011, 52, 6785-6792. 
Nirenberg S., Pandarinath C., Retinal prosthetic strategy with the capacity to restore normal vision, Proc Natl Acad Sci USA, 2012,109, 15012-15017.

Palanker D., Huie P., Vankov A., Aramant R., Seiler M., Fishman H., Marmor M., Blumenkranz M., Migration of retinal cells through a perforated membrane: implications for a high-resolution prosthesis. Invest Ophthalmol Vis Sci, 2004, 45, 3266-3270.

Perez Fornos A., Pittard A, Safran A.B., Pelizzone M., Simulation of artificial vision: IV. Visual information required to achieve simple pointing and manipulation tasks. Vision Res, 2008, 48, 1705-1718.

Rizzo J.F., 3rd, Wyatt J., Loewenstein J., Kelly S., Shire D., Methods and perceptual thresholds for short-term electrical stimulation of human retina with microelectrode arrays. Invest Ophthalmol Vis Sci, 2003a, 44, 5355-5361.

Rizzo J.F., 3rd, Wyatt J., Loewenstein J., Kelly S., Shire D., Perceptual efficacy of electrical stimulation of human retina with a microelectrode array during shortterm surgical trials. Invest Ophthalmol Vis Sci, 2003b, 44, 5362-5369.

Roska B., Werblin F., Vertical interactions across ten parallel, stacked representations in the mammalian retina. Nature, 2001, 410, 583-587.

Sommerhalder J., Oueghlani E., Bagnoud M., Leonards U., Safran A.B., Pelizzone M., Simulation of artificial vision: I. Eccentric reading of isolated words, and perceptual learning. Vision Res, 2003, 43, 269-283.

Sommerhalder J., Rappaz B., de Haller R., Fornos A.P., Safran A.B., Pelizzone M., Simulation of artificial vision: II. Eccentric reading of full-page text and the learning of this task. Vision Res, 2004, 44, 1693-1706.

Striem-Amit E., Cohen L., Dehaene S., Amedi A., Reading with sounds: sensory substitution selectively activates the visual word form area in the blind. Neuron, 2012, $76,640-652$.

Veraart C., Raftopoulos C., Mortimer J.T., Delbeke J., Pins D., Michaux G., Vanlierde A., Parrini S., WanetDefalque M.C., Visual sensations produced by optic nerve stimulation using an implanted self-sizing spiral cuff electrode. Brain Res, 1998, 813, 181-186.
Victor J.D., The dynamics of the cat retinal X cell centre. J Physiol, 1987, 386, 219-246.

Victor J.D., The dynamics of the cat retinal Y cell subunit. J Physiol, 1988, 405, 289-320.

Villegas-Perez M.P., Lawrence J.M., Vidal-Sanz M., Lavail M.M., Lund R.D., Ganglion cell loss in RCS rat retina: a result of compression of axons by contracting intraretinal vessels linked to the pigment epithelium. J Comp Neurol, 1998, 392, 58-77.

Wang L., Mathieson K., Kamins T.I., Loudin J.D., Galambos L., Goetz G., Sher A., Mandel Y., Huie P., Lavinsky D., Harris J.S., Palanker D.V., Photovoltaic retinal prosthesis: implant fabrication and performance. J Neur Eng, 2012, 9, 046014.

Wang S., Villegas-Perez M.P., Vidal-Sanz M., Lund R.D., Progressive optic axon dystrophy and vascular changes in rd mice. Invest Ophthalmol Vis Sci, 2000, 41, $537-545$.

Wilke R., Gabel V.P., Sachs H., Bartz Schmidt K.U., Gekeler F., Szurman P., Stett A., Wilhelm B., Peters T., Harscher A., Greppmaier U., Kibbel S., Benav H., Bruckmann A., Stingl K., Kusnyerik A., Zrenner E., Spatial resolution and perception of patterns mediated by a subretinal 16-electrode array in patients blinded by hereditary retinal dystrophies. Invest Ophthalmol Vis Sci, 2011, 52, 5995-6003.

Wilms M., Eckhorn R., Spatiotemporal receptive field properties of epiretinally recorded spikes and local electroretinograms in cats. BMC Neurosci, 2005, 6, 50.

Zeitz O., Keseru M., Hornig R., Richard G., Artificial sight: recent developments. Klin Monbl Augenheilkd, 2009, 226, 149-153.

Zrenner E., Will retinal implants restore vision? Science, 2002, 295, 1022-1025.

Zrenner E., Bartz-Schmidt K.U., Benav H., Besch D., Bruckmann A., Gabel V.P., Gekeler F., Greppmaier U., Harscher A., Kibbel S., Koch J., Kusnyerik A., Peters T., Stingl K., Sachs H., Stett A., Szurman P., Wilhelm B., Wilke R., Subretinal electronic chips allow blind patients to read letters and combine them to words. Proc Biol Sci, 2011, 278, 1489-1497. 